\title{
Immunohistochemical assessment of primary breast tumors and metachronous brain metastases, with particular regard to differences in the expression of biological markers and prognosis
}

\author{
YAWARA OMOTO ${ }^{1,3}$, MASAFUMI KUROSUMI ${ }^{1}$, YASUO HOZUMI ${ }^{3}$, HANAKO OBA $^{1}$, \\ KAORI KAWANOWA ${ }^{1}$, HIROYUKI TAKEI ${ }^{2}$ and YOSHIKAZU YASUDA ${ }^{4}$ \\ Departments of ${ }^{1}$ Pathology, and ${ }^{2}$ Breast Surgery, Saitama Cancer Center, Saitama 362-0806; \\ Divisions of ${ }^{3}$ Breast and General Surgery, and ${ }^{4}$ Gastrointestinal Surgery, Jichi Medical University, Tochigi 329-0498, Japan
}

Received April 8, 2010; Accepted June 7, 2010

DOI: 10.3892/etm_00000088

\begin{abstract}
In this study, we conducted an immunohistochemical analysis of primary breast tumors and metachronous brain metastases to compare the differences in the expression of biological markers between the two. Carcinoma tissues from primary breast tumors and metachronous brain metastases collected from 21 patients were examined immunohistochemically for the expression status of the estrogen receptor (ER), progesterone receptor $(\mathrm{PgR})$, human epidermal growth factor receptor (HER)-2, Ki-67, bcl-2 and p53, and the results were compared. In addition, the relationships between the expression of these factors and prognosis were evaluated. There were no significant differences in the frequencies of ER-, PgR-, HER-2-, bcl-2- or p53-positivity between the primary breast tumors and metachronous brain metastases. While the Ki-67 labeling index (LI) was high in both the primary breast tumors and brain metastases, it was significantly higher in the brain metastases than in the corresponding primary breast tumors $(\mathrm{P}=0.003)$. With regard to the prognosis, breast cancer patients who showed ER-positivity in the primary tumors showed significantly longer survival after primary diagnosis $(\mathrm{P}=0.0076)$. Furthermore, breast cancer patients who exhibited ER-positivity, bcl-2-positivity or p53-negativity in the primary tumors showed significantly longer intervals from primary diagnosis to the detection of the brain metastases. Of all the markers, only the Ki-67 LI was significantly higher in the brain metastases than in the primary breast tumors. We confirmed that the tumor characteristics were worse in the metachronous brain metastases.
\end{abstract}

Correspondence to: Dr Masafumi Kurosumi, Department of Pathology, Saitama Cancer Center, 818 Komuro, Ina-machi, Kitaadachi-gun, Saitama 362-0806, Japan

E-mail: mkurosumi@cancer-c.pref.saitama.jp

Key words: breast cancer, brain metastasis, immunohistochemical expression, biological markers, prognosis

\section{Introduction}

Brain metastasis occurs frequently in women with advanced breast cancer. However, early breast cancer patients developing metastasis to the brain as the first site of recurrence are occasionally encountered. Certain studies suggest that only $10-16 \%$ of patients with metastatic breast cancer have clinically apparent brain metastases, and autopsy series have revealed an incidence of brain metastasis of $30 \%$ in breast cancer patients (1). However, it is a matter of great concern that few effective treatments are available for the treatment of clinically apparent brain metastases. It is well known that almost none of the anticancer agents are effective against brain metastases due to the presence of the blood-brain barrier. Therefore, the main treatment method for brain metastases is either radiotherapy or surgical therapy. However, even after these treatments, the mean 1-year survival rate is estimated to be less than $20 \%(2,3)$. Therefore, more effective treatments for brain metastases are required.

Some clinicians have suggested that the incidence of brain metastasis in breast cancer patients has increased following the introduction of trastuzumab, a humanized recombinant monoclonal antibody against human epidermal growth factor receptor (HER)-2/neu, although the cause/effect relationship remains unclear and controversial. A number of possibilities have, however, been proposed: i) HER-2-positive breast tumors are more likely to metastasize to the brain; ii) trastuzumab may promote the development of brain metastasis; iii) prolongation of the survival period owing to the efficacy of trastuzumab against visceral metastases results in an increase in the incidence of brain metastasis. If trastuzumab was not effective against only brain metastases, the blood-brain barrier could play an important role in the development of brain metastases. However, we think that changes in the biological features of the tumors also contribute to the development of brain metastasis.

The HER-2 expression status is a predictive factor for the efficacy of trastuzumab and also a prognostic factor in breast cancer patients. In general, it is well known that breast cancer patients with HER-2/neu overexpression have a poor prognosis. The estrogen receptor (ER) and progesterone 
receptor $(\mathrm{PgR})$ are the main hormone receptors expressed in breast cancers, and the expression status of these receptors is the best predictive factor for hormonal therapy. Moreover, ER- and PgR-negative breast cancers show poor prognosis and are more likely associated with brain metastasis (4-8). p53 and bcl-2 are apoptosis-related factors, and $\mathrm{Ki}-67$ is a proliferation-related factor. Evaluating the differences in the expression of these factors between primary breast tumors and brain metastases is very important in order to elucidate the mechanism of development of brain metastasis.

In the present study, we comparatively evaluated the expression of these factors by immunohistochemistry in primary breast tumors and metachronous brain metastases. Moreover, the relationships between the expression of these factors and prognosis were also examined.

\section{Materials and methods}

Patients and tissue samples. This study was conducted on 21 patients who were diagnosed as having breast cancer and who underwent breast surgery at the Saitama Cancer Center, Japan, between 1984 and 2004. All 21 patients developed metachronous brain metastasis and underwent craniotomy and tumor resection, followed by whole-brain radiotherapy. None of the patients had received trastuzumab before the onset of the brain metastasis. For all of the 21 patients, specimens of the primary breast and metastatic brain tumors were available for the study. All of the resected tissues were fixed in $10 \%$ formalin solution, embedded in paraffin and stained with $\mathrm{H} \& \mathrm{E}$ for routine histopathologic examination. The expression of ER, PgR and HER-2 in the primary breast cancers was compared to that in usual invasive breast cancers. These usual breast cancers consisted of 439 (for ER and PgR) or 586 (for HER-2) consecutive tumor samples collected at the Saitama Cancer Center between 2003 and 2004.

Immunohistochemistry. Immunohistochemical staining was performed on the resected tissues. Sections were deparaffinized with xylene and dehydrated through a graded series of ethanol. To enhance the antigenicity, the sections were immersed in $10 \mathrm{mmol} / \mathrm{l}$ citrate buffer ( $\mathrm{pH} \mathrm{6.0)}$ and autoclaved. Endogenous peroxidase activity was blocked with $0.3 \%$ hydrogen peroxide in methanol. Sections were then incubated for $1 \mathrm{~h}$ with monoclonal antibodies against ER (1:50), PgR (1:50), p53 (1:100), bcl-2 (1:40), Ki-67 (1:50) or polyclonal antibody against HER-2/neu (1:1,600) (all from Dako, Glostrup, Denmark). Incubation with a secondary antibody (peroxidaselabeled Envision polymer reagent; Dako) was performed for $45 \mathrm{~min}$ at room temperature. After visualization of the reaction complexes with $0.05 \%$ 3,3'-diaminobenzidine tetrahydrochloride and $0.03 \%$ hydrogen peroxide in $50 \mathrm{mmol} / 1$ Tris-HCL buffer ( $\mathrm{pH}$ 7.6), the sections were examined under a light microscope.

Specimen analysis. Two investigators performed the microscopic examinations independently. Immunoreactivity was quantified by evaluating a minimum of 1,000 carcinoma cells in the histologic sections examined in randomly selected fields under a high-powered objective. Strong to moderate immunostaining was considered as positive staining and faint
Table I. Demographic data

\begin{tabular}{lc}
\hline Characteristics & $\mathrm{n}=21$ \\
\hline Age (years) & \\
Median at $1^{0}$ Dx & 47 \\
Range at $1^{0}$ Dx & $33-69$ \\
Average TTD from $1^{0}$ Dx (months) & 56.0 \\
Average TTD from Met (months) & 12.0 \\
Average TTM from $1^{0}$ Dx (months) & 44.5 \\
Cause of death (n=17) & \\
CNS/non-CNS & $11 / 6$ \\
No. of Met & $8 / 13$ \\
Single/multiple & \\
\hline
\end{tabular}

$1^{0} \mathrm{Dx}$, primary diagnosis; TTD, time to death; Met, brain metastasis; TTM, time to the development of brain metastasis; CNS, central nervous system.

to no staining was considered as negative staining. The results for ER, PgR and p53 were considered positive when $>10 \%$ of the nuclei of the carcinoma cells showed positive staining for the respective markers. As for bcl-2, the expression status was considered positive when $>25 \%$ of the cytoplasm of the carcinoma cells showed positive staining. The Ki-67 labeling index (LI) was calculated as the percentage of carcinoma cells with Ki-67-positive nuclei. A Ki-67 LI $\geq 20 \%$ was considered to be a high value of the index. For the evaluation of HER-2/ neu expression, semi-quantitative analysis was performed according to the scoring guidelines laid down in the HercepTest $^{\mathrm{TM}}$ (Dako) instruction guide. In brief, the specimens were scored as follows: 0 , no staining or membrane staining in $<10 \%$ of the tumor cells; $1+$, faint or barely perceptible membrane staining in $>10 \%$ of the tumor cells; $2+$, weak to moderate complete membrane staining in $>10 \%$ of the tumor cells; $3+$, strong complete membrane staining in $>30 \%$ of the tumor cells. Scores of 0 and $1+$ represented a negative result for HER-2/neu overexpression, whereas scores of $2+$ and $3+$ were considered to represent HER-2/neu overexpression. Only the membrane staining intensity and patterns were evaluated as laid down in the guideline.

Statistical analysis. The $\chi^{2}$ analysis was used for univariate categorical variable analysis. In relation to ER, PgR, HER-2, bcl-2 and p53 expression, survival curves were calculated using the Kaplan-Meier method and compared by the log-rank test. The Ki-67 LI in the primary tumors was compared to that in the brain metastases using a paired t-test. A P-value $<0.05$ was considered as denoting statistical significance.

\section{Results}

Clinical and pathological characteristics. All 21 patients were female, with a median age of 47 years (range 33-69) at the time of diagnosis of the primary breast cancer. Of the 21 patients, 1 had received pre-operative chemotherapy prior to the breast surgery. Thirteen patients had received at least 
Table II. Positive expression rate and conversion of expression.

\begin{tabular}{|c|c|c|c|c|c|c|}
\hline & \multicolumn{2}{|c|}{ Positive expression rate } & \multirow[b]{2}{*}{$\mathrm{PCC}$} & \multirow[b]{2}{*}{$\mathrm{NCC}$} & \multirow[b]{2}{*}{ CR } & \multirow[b]{2}{*}{ NCR } \\
\hline & Primary & Brain & & & & \\
\hline ER & $43 \% \quad(9 / 21)$ & $43 \% \quad(9 / 21)$ & 2 & 2 & $81 \%(17 / 21)$ & $22 \% \quad(2 / 9)$ \\
\hline PgR & $29 \% \quad(6 / 21)$ & $19 \% \quad(4 / 21)$ & 1 & 3 & $81 \%(17 / 21)$ & $50 \% \quad(3 / 6)$ \\
\hline HER-2 & $33 \% \quad(7 / 21)$ & $43 \% \quad(9 / 21)$ & 3 & 1 & $81 \%(17 / 21)$ & $14 \% \quad(1 / 7)$ \\
\hline p53 & $33 \% \quad(7 / 21)$ & $48 \%(10 / 21)$ & 3 & 0 & $86 \%(18 / 21)$ & $0 \% \quad(0 / 7)$ \\
\hline bcl-2 & $57 \%(12 / 21)$ & $43 \% \quad(9 / 21)$ & 1 & 4 & $76 \%(16 / 21)$ & $33 \%(4 / 12)$ \\
\hline
\end{tabular}

PCC, positive conversion cases; NCC, negative conversion cases; CR, concordance rate; NCR, negative conversion rate.
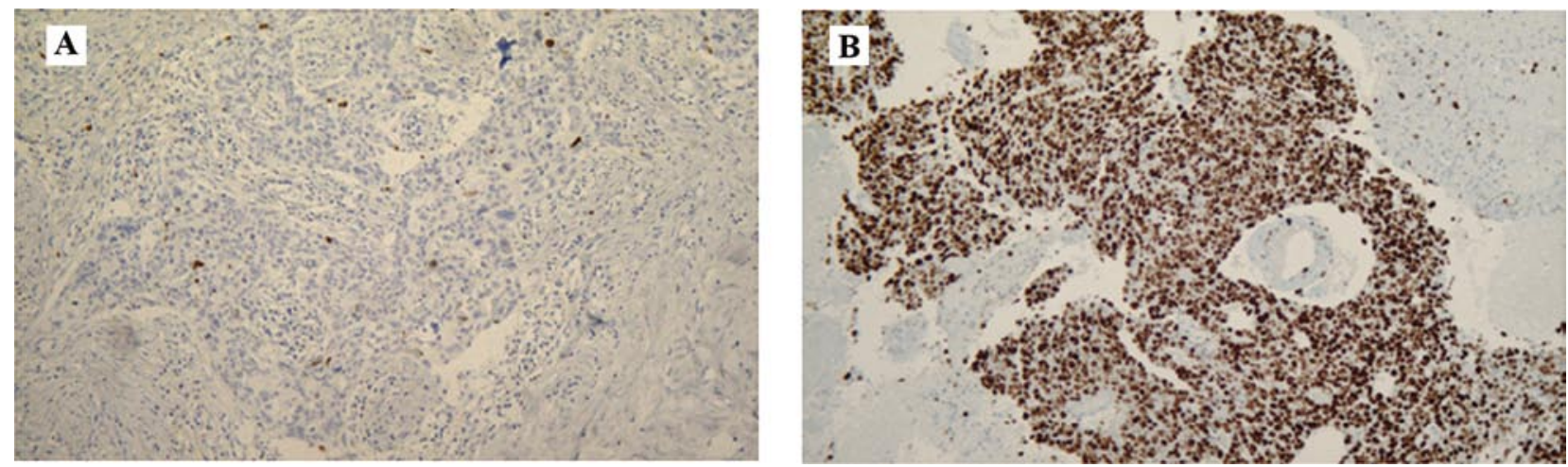

Figure 1. A case showing a markedly higher Ki-67 LI value in the brain metastasis (B) than that in the primary breast tumor (A). Magnification x100.

one form of postoperative hormonal therapy, 16 had received at least one form of postoperative chemotherapy and 10 had received both after the breast surgery. Only 1 patient had received trastuzumab after the brain surgery. The average interval from primary diagnosis to death was 56 months, whereas the average interval from the time of development of brain metastasis to death was 12 months. Death was ascribed to brain metastasis in 11 of the 17 patients and to non-central nervous system causes in 6 of the 17 patients (Table I).

Characteristics of the primary tumors. The primary tumors were positive for ER, PgR, HER-2, p53 and bcl-2 expression in $43 \%(9 / 21), 29 \%(6 / 21), 33 \%(7 / 21), 33 \%(7 / 21)$ and $57 \%$ $(12 / 21)$ of the cases, respectively. The Ki-67 LI was high in $67 \%(14 / 21)$ of the primary tumors and not high in the remaining $33 \%$ cases $(7 / 21)$, with a mean \pm SD percentage of immunoreactive cells of $25.6 \pm 14.6 \%$. The percentages of ER- and/or PgR-positive cases in the primary tumors were significantly lower than the corresponding percentages in the usual invasive breast cancers (ER, $\mathrm{P}=0.0002$; PgR, $\mathrm{P}=0.00018)$. However, there was no significant difference in the percentage of HER-2-positive specimens between the primary tumors and usual invasive breast cancers $(\mathrm{P}=0.64)$.

Characteristics of the brain metastases. The brain metastases were positive for ER, PgR, HER-2, p53 and bcl-2 expression in $43 \%(9 / 21), 19 \%(4 / 21), 43 \%(9 / 21), 48 \%(10 / 21)$ and $43 \%$ (9/21) of the cases, respectively. The Ki-67 LI was high in $95 \%$ (20/21) of the brain metastases and not high in the remaining

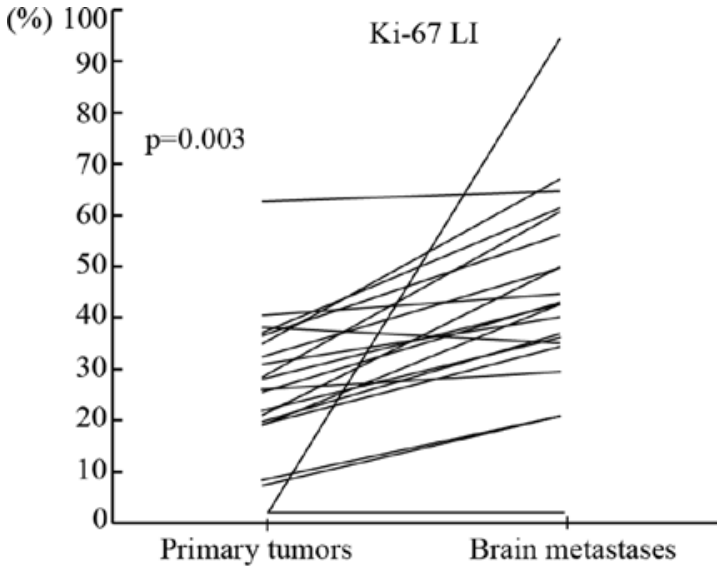

Figure 2. Comparison of the Ki-67 LI between primary breast tumors and metachronous brain metastases. The Ki-67 LI values were significantly higher in the brain metastases than those in the primary breast tumors $(\mathrm{P}=0.003$, paired t-test).

$5 \%$ cases $(1 / 21)$, with a mean \pm SD percentage of immunoreactive cells of $44.3 \pm 19.8 \%$.

Comparison of the expression in the primary tumors and brain metastases. The concordance rate between the primary tumors and brain metastases was high for p53 [86\% (18/21)], ER, PgR and HER-2 [81\% (17/21) for all three]. The negativeconversion rates for ER and PgR were 22\% (2/9) and 50\% $(3 / 6)$, respectively (Table II); all of these patients had received 

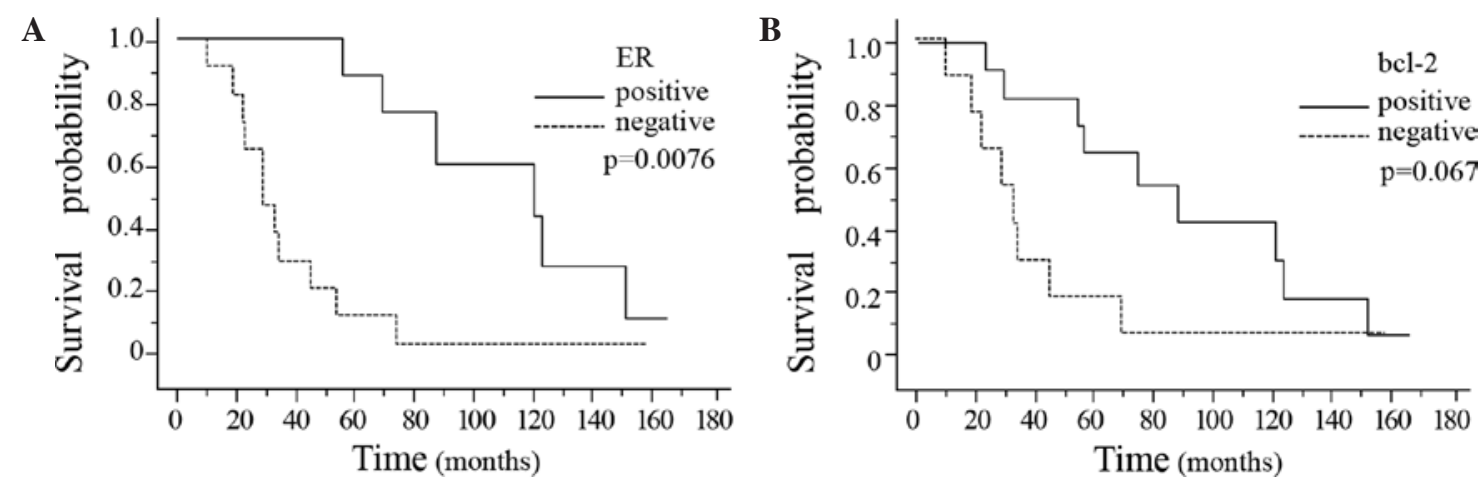

Figure 3. Kaplan-Meier survival curves were plotted based on ER and bcl-2 expression in the primary breast tumors. The interval from the primary diagnosis to death was significantly longer in the patients with positive ER expression in the primary breast tumor $(\mathrm{A}, \mathrm{P}=0.0076$; log-rank test). A similar tendency was noted in relation to the patients showing positive bcl- 2 expression in the primary breast tumor $(\mathrm{B}, \mathrm{P}=0.067$; log-rank test).

postoperative hormonal therapy after breast surgery. The Ki-67 LI was significantly higher in the brain metastases than that in the primary tumors ( $\mathrm{P}=0.003$ ) (Figs. 1 and 2).

Survival data. The time from primary diagnosis to death was significantly longer in the breast cancer patients showing positive ER expression in the primary breast cancer tissue $(\mathrm{P}=0.0076)$ (Fig. 3A). Moreover, the interval from primary diagnosis to death also tended to be longer in the patients showing positive bcl-2 expression in the primary breast cancer tissue as compared to that in the patients showing negative bcl-2 expression in the primary breast tumor $(\mathrm{P}=0.067)$ (Fig. 3B). There were no significant differences in the interval from the diagnosis of brain metastasis to death associated with the presence/absence of ER, PgR, HER-2, p53 or bcl-2 in either the primary breast tumors or the brain metastases (Table III). The interval from primary diagnosis to the development of brain metastasis was significantly longer in the breast cancer patients showing ER-positivity, p53-negativity or bcl-2-positivity in the primary breast cancer tissue (Fig. 4A-C). Of the three markers, bcl-2 was the most sensitive prognostic marker $(\mathrm{P}<0.0001)$. The interval from primary diagnosis to the detection of brain metastasis tended to be longer in patients showing a Ki-67 LI of $<20 \%$ in the breast cancer tissue (Fig. 4D).

\section{Discussion}

In the present study, we demonstrated a striking increase in the $\mathrm{Ki}-67 \mathrm{LI}$ in the metachronous brain metastases as compared to that in the corresponding primary breast tumors. We clarified the differences in the characteristics between the primary breast cancer and the metachronous brain metastases in this study. The Ki-67 nuclear antigen is known to be present during the G1, S, G2 and M, but not the G0, phases of the cell cycle in continuously proliferating cells. Therefore, immunohistochemical staining with an antibody against Ki-67 serves as a useful means to determine the cell proliferative activity (9). It has been reported that breast cancer patients with a high Ki-67 LI in the primary tumor are likely to have a poor prognosis (10-15). Some investigators have demonstrated that a high Ki-67 LI is a predictive marker of a better response to chemotherapy in breast cancer patients (16-18), although the precise relationship between the Ki-67 LI and the efficacy of chemotherapy is still controversial (19). This may be partially attributed to the heterogeneity of Ki-67 expression. Shabani et al, using a small sample size, also showed significantly higher values of the $\mathrm{Ki}-67 \mathrm{LI}$ in brain metastases than in primary cancers (the origins of various origins) (20). Moreover, some investigators have reported that the Ki-67 LI was significantly higher in metastatic axillary lymph nodes than in the corresponding primary breast tumors $(21,22)$. The differences in the Ki-67 LI between primary breast tumors and metastatic tumors have not yet been clarified in relation to other organs. However, it could be reasonably presumed that the Ki-67 LI would be higher in metastatic tissues than that in the primary tumors. In the present study, we confirmed that the Ki-67 LI was significantly higher in the brain metastases than in the primary breast cancers, despite the existence of heterogeneity.

As compared to the case in usual invasive breast cancer, the percentage of ER-and/or PgR-positive cases was significantly lower in the patients with metachronous brain metastases, which was consistent with previous reports (4-8). However, the percentage of HER-2-positive cases was not significantly different between these two groups. It has been reported that $42-48 \%$ of breast tumors associated with metachronous brain metastases show positive HER-2 expression $(23,24)$. Thus, our result of $33 \%$ seems to be relatively low; we speculated that this may simply be due to the small sample size in our study.

In the present study, the concordance rates between the primary breast tumors and the brain metastases were high (more than 76\%) for ER, PgR, HER-2, p53 and bcl-2 expression. Of the 21 primary breast tumors, 9 cases were positive for ER and 6 for PgR. The negative conversion rate was $22 \%$ (2/9) for ER and 50\% (3/6) for PgR; all patients that showed negative ER and/or PgR conversions had received hormonal therapy after the breast surgery. These results raise the possibility of a relationship between negative conversion for hormone receptor expression and the efficacy of hormonal therapy. However, this phenomenon (negative conversion) does not always occur after hormonal therapy. On the other hand, negative ER and/or PgR conversions have also been found to be frequently induced by pre-operative hormonal therapy (25). 
Table III. Survival data (mean time to death in months).

\begin{tabular}{|c|c|c|c|c|c|c|c|c|c|}
\hline \multicolumn{2}{|c|}{ ER } & \multicolumn{2}{|c|}{$\operatorname{PgR}$} & \multicolumn{2}{|c|}{ HER-2 } & \multicolumn{2}{|c|}{ p53 } & \multicolumn{2}{|c|}{ bcl-2 } \\
\hline+ & - & + & - & + & - & + & - & + & - \\
\hline
\end{tabular}

Staining, breast

Survival from diagnosis of

112.2

$$
92.3 \quad 63.3
$$

$43.7 \quad 79.4$

39.1

84.4

92.8

36.7

primary

0.0076

0.18

$\begin{array}{llll}37.3 & 11.3 & 13.5 & 24.0\end{array}$
0.38
$26.5 \quad 21.6$

0.24

0.067

Survival from diagnosis of brain metastasis $(n=21)$

P-value

0.13

0.88

$11.0 \quad 27.4$

26.6

Staining, brain

Survival from diagnosis of brain metastasis $(n=21)$

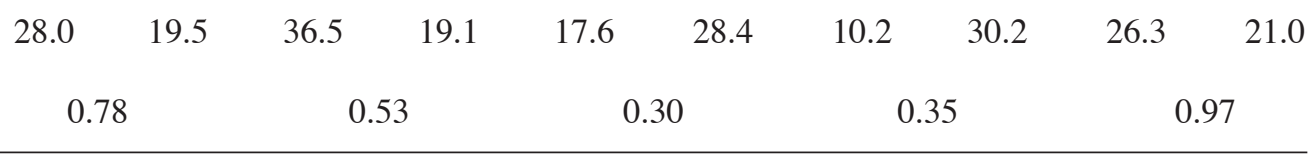

A

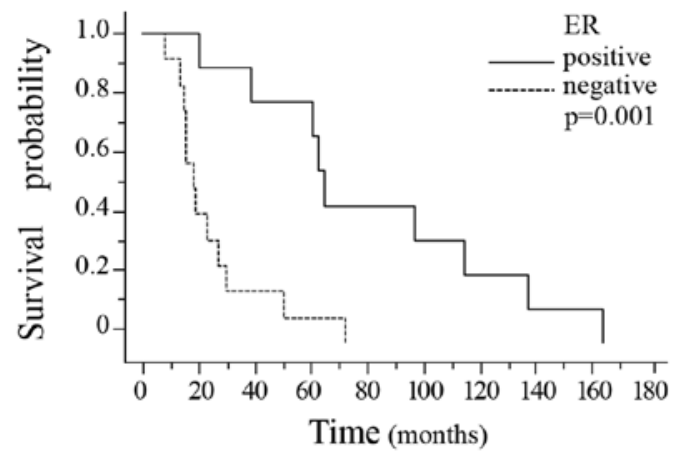

$\mathbf{C}$

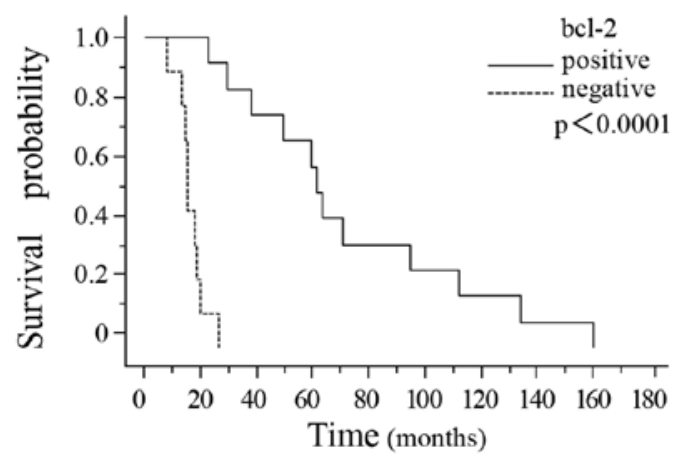

B

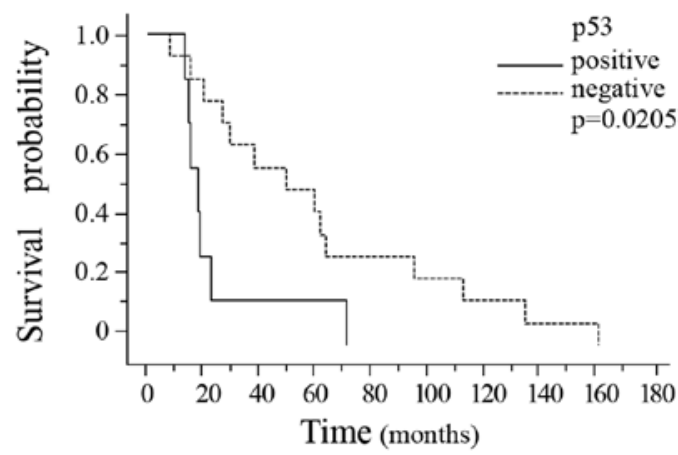

D

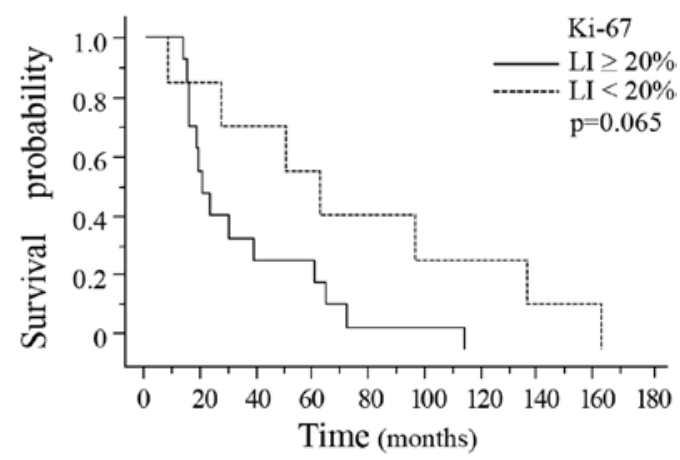

Figure 4. Kaplan-Meier survival curves were plotted based on the ER, p53 and bcl-2 expression and the Ki-67 LI in the primary breast tumors. A significantly longer interval from the primary diagnosis to the development of brain metastasis was noted in the patients showing positive ER, negative p53 or positive bcl-2 expression in the primary breast tumor (A, $\mathrm{P}=0.001 ; \mathrm{B}, \mathrm{P}=0.0205 ; \mathrm{C}, \mathrm{P}<0.0001 ; \log$-rank test). The interval from primary diagnosis to the development of brain metastasis tended to be longer in the patients with a Ki-67 LI of $<20 \%$ in the primary breast tumor (D, P=0.065; log-rank test).

Although the mechanisms underlying these phenomena have yet to be fully elucidated, negative conversion of hormone receptor expression appears to be an important factor in the resistance to hormonal therapies.

The percentage of p53-positive cases was higher by $15 \%$ in the brain metastases than in the primary breast tumors; that is, 3 of the 14 p53-negative primary breast tumors showed positive conversion in the brain metastases. Some investigators have suggested that there is a considerable difference in the rate of p53 abnormalities between immunohistochemical and mutational analysis (26-28). Immunohistochemical analysis detects mutated p53 protein, but not wild-type p53. This is thought to be due to the longer half-life of mutated p53 protein. Failure of apoptosis is attributed to the p53 mutation. Thus, the detection of p53 protein is considered to be associated with worsening tumor characteristics. On the other hand, although bcl-2 is generally considered to be an anti-apoptotic protein, most investigators agree that bcl-2-positive breast cancers show good responses to hormonal therapy. Moreover, bcl-2 expression has been reported to be associated with favor- 
able histopathological features and positive clinical outcomes $(29,30)$. This may seem paradoxical, but we consider that the presence of the bcl-2 protein sustains the estrogen-dependent proliferative organization of the tumors. The percentage of bcl-2-positive cases was lower by $14 \%$ in the brain metastases than in the primary breast tumors; that is, 4 of the 12 bcl-2positive primary breast tumors showed negative conversion in the brain metastases, and 1 of the 9 bcl-2-negative primary breast tumors showed positive conversion in the brain metastases. Positive hormone receptor expression is a favorable prognostic factor in breast cancer patients; therefore, the decrease in the percentage of bcl-2-positive cases in the brain metastases is not likely to reflect a favorable prognosis. In this study, the increase in the number of p53-positive cases and Ki-67-positive cells, and the decrease in the number of bcl-2positive cases in the brain metastases suggest a worsening of the tumor characteristics in brain metastases.

In our study, the interval from primary diagnosis to death was significantly longer in the breast cancer patients who showed positive ER expression in the primary breast cancer tissue. This tendency was also observed for bcl-2 expression. Furthermore, the interval from primary diagnosis to the detection of brain metastasis was significantly longer in the breast cancer patients showing ER-positivity, p53-negativity or bcl2positivity in the primary breast cancer tissue. In relation to ER and bcl-2, the prolongation of the interval from primary diagnosis to the detection of brain metastasis contributed to the prolongation of the interval from primary diagnosis to death. From this point of view, ER and bcl-2 are important prognostic factors in these patients.

We also examined the correlation between the Ki-67 LI and prognosis. There was no relationship between the $\mathrm{Ki}-67 \mathrm{LI}$ in breast cancer tissue and the interval from primary diagnosis to death. Moreover, there was also no relationship between the $\mathrm{Ki}-67 \mathrm{LI}$ in the brain metastases and the interval from the detection of brain metastasis to death. Caly et al reported the Ki-67 $\mathrm{LI}$ as a prognostic factor in terms of both disease-free survival and overall survival (OS) based on a study of 257 breast cancer patients (15). In our study, the absence of a relationship between the Ki-67 LI in the breast cancer tissue and the OS could be attributable to the small sample size. However, the interval from primary diagnosis to the detection of brain metastasis tended to be longer in breast cancer patients showing a Ki-67 LI of less than $20 \%$ in the breast cancer tissue.

In conclusion, we comparatively evaluated the immunohistochemical expression profiles between primary breast tumors and brain metastases in this study. There were no significant differences in the expression of ER, PgR, HER-2, p53 or bcl-2 between the two tissues. However, the Ki-67 LI was significantly higher in the brain metastases than in the primary breast tumors. Furthermore, a high Ki-67 LI in the primary breast tumor was associated with a shorter interval from the primary diagnosis to the development of brain metastasis. These results suggest that the tumor characteristics are worse in the brain metastases. In addition, ER and/or bcl-2 expression was shown to be an important prognostic factor in breast cancer patients with metachronous brain metastases. Further studies in larger samples are required to understand the relationship between the immunohistochemical expression profiles of primary breast tumor and brain metastases.

\section{Acknowledgements}

The authors acknowledge financial support by a grant from the 'Brain Metastasis of Breast Cancer' Research Group (Director, Dr Michihide Mitsumori) of the Japanese Breast Cancer Society.

\section{References}

1. Lin NU, Bellon JR and Winer EP: CNS metastases in breast cancer. J Clin Oncol 22: 3608-3617, 2004.

2. Shaffrey ME, Mut M, Asher AL, Burri SH, Chahlavi A, Chang SM, Farace E, Fiveash JB, Lang FF, Lopes MB, Markert JM, Schiff D, Siomin V, Tatter SB and Voqelbaum MA: Brain metastasis. Curr Probl Surg 41: 665-741, 2004.

3. Engel J, Eckel R, Aydemir U, Aydemir S, Kerr J, Schlesinger-Raab A, Dirschedl P and Holzel D: Determinants and prognoses of locoregional and distant progression in breast cancer. Int J Radiat Oncol Biol Phys 55: 1186-1195, 2003.

4. Hicks DG, Short SM, Prescott NL, Tarr SM, Coleman KA, Yoder BJ, Crowe JP, Choueiri TK, Dawson AE, Butt GT, Tubbs RR, Casey G and Weil RJ: Breast cancers with brain metastases are more likely to be estrogen receptor negative, express the basal cytokeratin CK5/6, and overexpress HER2 or EGFR. Am J Surg Pathol 30: 1097-1103, 2006.

5. Ryberg M, Nielsen D, Osterlind K, Andersen PK, Skovsgaard T and Dombernowsky P: Predictors of central nervous system metastasis in patients with metastatic breast cancer: A competing risk analysis of 579 patients treated with epirubicin-based chemotherapy. Breast Cancer Res Treat 91: 217-225, 2005.

6. Evans AJ, James JJ, Cornford EJ, Chan SY, Burrell HC, Pinder SE, Gutteridge E, Robertson JF, Hornbuckle J and Cheung KL: Brain metastasis from breast cancer: identification of a high-risk group. Clin Oncol 16: 345-349, 2004.

7. Slimane K, Andre F, Delaloge S, Dunant A, Perez A, Grenier J, Massard $C$ and Spielmann M: Risk factors for brain relapse in patients with metastatic breast cancer. Ann Oncol 15: 1640-1644, 2004.

8. Maki D and Grossman RI: Patterns of disease spread in metastatic breast carcinoma: influence of estrogen and progesterone receptor status. AJNR Am J Neuroradiol 21: 1064-1066, 2000.

9. Gerdes J, Lemke H, Baisch H, Wacker HH, Schwab U and Stein H: Cell cycle analysis of a cell proliferation-associated human nuclear antigen defined by the monoclonal antibody Ki-67. J Immunol 133: 1710-1715, 1984.

10. Molino A, Micciolo R, Turazza M, Bonetti F, Piubello Q, Bonetti A, Nortilli R, Pelosi G and Cetto GL: Ki-67 immunostaining in 322 primary breast cancers: associations with clinical and pathological variables and prognosis. Int J Cancer 74: 433-437, 1997.

11. Railo M, Lundin J, Haglund C, von Smitten K, von Boguslawsky K and Nordling S: Ki-67, p53, Er-receptors, ploidy and S-phase as prognostic factors in $\mathrm{T} 1$ node negative breast cancer. Acta Oncol 36: 369-374, 1997.

12. Pierga JY, Leroyer A, Viehl P, Mosseri V, Chvillard S and Magdelenat $\mathrm{H}$ : Long term prognostic value of growth fraction determination by Ki-67 immunostaining in primary operable breast cancer. Breast Cancer Res Treat 37: 57-64, 1996.

13. Railo M, Nordling S, von Boguslawsky $K$, Leivonen $M$, Kyllonen L and von Smitten K: Prognostic value of Ki-67 immunolabelling in primary operable breast cancer. Br J Cancer 68: 579-583, 1993.

14. Veronese SM, Gambacorta M, Gottardi O, Scanzi F, Ferrari M and Lampertico P: Proliferation index as a prognostic marker in breast cancer. Cancer 71: 3926-3931, 1993.

15. Caly M, Genin P, Al Ghuzlan A, Elie C, Freneaux P, Klijanienko J, Rosty C, Sigal-Zafrani B, Vincent-Salomoni A, Douggaz A, Zidane $M$ and Sastre-Garau X: Analysis of correlation between mitotic index, MIB1 score and S-phase fraction as proliferation markers in invasive breast carcinoma. Methodological aspects and prognostic value in a series of 257 cases. Anticancer Res 24: 3283-3288, 2004.

16. Petit T, Wilt M, Velten M, Millon R, Rodier JF, Borel C, Mors R, Haegele P, Eber M and Ghnassia JP: Comparative value of tumour grade hormonal receptors, Ki-67, HER-2 and topoisomerase II alpha status as predictive markers in breast cancer patients treated with neoadjuvant anthracycline-based chemotherapy. Eur J Cancer 40: 205-211, 2004. 
17. Bonetti A, Zaninelli M, Rodella S, Molino A, Sperotto L, Piubello Q, Bonetti F, Nortilli R, Turazza M and Cetto GL: Tumor proliferative activity and response to first-line chemotherapy in advanced breast carcinoma. Breast Cancer Res Treat 38: 289-297, 1996.

18. MacGrogan G, Mauriac L, Durand M, Bonichon F, Trojani M, De Mascarel I and Coindre JM: Primary chemotherapy in breast invasive carcinoma: predictive value of the immunohistochemical detection of hormonal receptors, p53, c-erbB-2, MiB1, pS2 and GST pi. Br J Cancer 74: 1458-1465, 1996.

19. Aas T, Geisler S, Eide GE, Haugen DF, Varhaug JE, Bassoe AM Thorsen T, Berntsen H, Borresen-Dale AL, Akslen LA and Lonning PE: Predictive value of tumor cell proliferation in locally advanced breast cancer treated with neoadjuvant chemotherapy. Eur J Cancer 39: 438-446, 2003.

20. Shabani HK, Kitange G, Tsunoda K, Anda T, Tokunaga Y, Shibata S, Kaminogo M, Hayashi T, Ayabe H and Iseki M: Immunohistochemical expression of E-cadherin in metastatic brain tumors. Brain Tumor Pathol 20: 7-12, 2003.

21. Buxant F, Anaf V, Simon P, Favt I and Noel JC: Ki-67 immunostaining activity is higher in positive axillary lymph nodes than in the primary breast tumor. Breast Cancer Res Treat 75: 1-3, 2002.

22. Cabibi D, Mustacchio V, Martorana A, Tripodo C, Campione M, Calascibetta A, Sanguedolce R and Aragona F: Lymph node metastases displaying lower Ki-67 immunostaining activity than the primary breast cancer. Anticancer Res 26: 4357-4360, 2006.

23. Lear-Kaul KC, Yoon HR, Kleinschmidt-DeMasters BK, McGavran L and Singh M: HER-2/neu status in breast cancer metastases to central nervous system. Arch Pathol Lab Med 127: 1451-1457, 2003.
24. Fuchs IB, Loebbecke M, Buhler H, Stoltenburg-Didinger G, Heine B, Lichtenegger W and Schaller G: HER2 in brain metastases: issues of concordance, survival, and treatment. J Clin Oncol 20: 4130-4133, 2002.

25. Ellis MJ, Coop A, Singh B, Tao Y, Llombart-Cussac A, Janicke F, Mauriac L, Quebe-Fehling E, Chaudri-Ross HA, Evans DB and Miller WR: Letrozole inhibits tumor proliferation more effectively than tamoxifen independent of HER1/2 expression status. Cancer Res 63: 6523-6531, 2003.

26. Dunn JM, Hastrich DJ, Newcomb P, Webb JCJ, Maitland NJ and Farndon JR: Correlation between p53 mutations and antibody staining in breast carcinoma. Br J Surg 80: 1410-1412, 1993.

27. Thompson AM, Anderson TJ, Condie A, Prosser J, Chetty U, Carter DC, Evans HJ and Steel CM: p53 allele losses, mutations and expression in breast cancer and their relationship to clinicopathological parameters. Int J Cancer 50: 528-532, 1992.

28. Borrensen AL, Hovig E, Smith-Sorensen B, Malkin D, Lystad S, Andersen TI, Nesland JM, Isselbacher KJ and Friend SH: Constant denaturation gel electrophoresis (CDGE) as a rapid screening technique for 553 mutations. Proc Natl Acad Sci USA 88: 8405-8409, 1991.

29. Binder C, Marx D, Overhoff R, Binder L, Schauer A and Hiddemann W: Bcl-2 protein expression in breast cancer in relation to established prognostic factors and other clinicopathological variables. Ann Oncol 6: 1005-1010, 1995.

30. Gee JM, Robertson JF, Ellis IO, Willsher P, McClelland RA, Hoyle HB, Kyme SR, Finlay P, Blamey RW and Nicholson RI: Immunocytochemical localization of bcl-2 protein in human breast cancers and its relationship to a series of prognostic markers and response to endocrine therapy. Int J Cancer 59: 619-628, 1994. 